\title{
IMPACTS DES ÉVOLUTIONS DÉMOGRAPHIQUES ET CLIMATIQUES SUR LA RÉPARTITION SPATIALE DES HOMMES, DES TSÉ-TSÉ ET DES TRYPANOSOMOSES eN AfRIQUE DE L'OUEST ${ }^{1}$
}

\author{
COURTIN F.*, SIDIBÉ I.**, ROUAMBA J.**, JAMONNEAU V.*, GOURO A.** \& SOLANO P.*
}

Summary: POPULATION GROWTH AND GLOBAL WARMING: IMPACTS ON TSETSE AND TRYPANOSOMOSES IN WEST AFRICA

Demographic evolution, climatic change and economical development that happened in West Africa during the XXth century had a lot of consequences on human settlement and landscape. These changes have in turn an impact on the pathogenic system of human and animal trypanosomoses. Since last century, the northern tsetse distribution limit has shifted towards the south, probably due to a decrease in rainfall combined to the impact of human pressure. Sleeping sickness (SS) foci have also shiffed from the savannah areas (where there is no more SS) to the forest and mangrove areas of West Africa, but animal trypanosomoses are still present in savannah. We show a decrease of tsetse of the morsitans group as a result of an increase of human densities. On the opposite, tsetse species like Glossina palpalis adapt to high human densities and are found in the biggest urban centres of West Africa. There is a need to promote multidisciplinary studies on this demographic-climatic-vector borne disease topic, especially in Africa to be able to define future areas of presence/absence of these diseases in order to help continental plans of control that have recently begun.

KEY WORDS : population growth, global warming, West Africa, impact, tsetse flies, trypanosomosis.

MOTS CLÉS : démographie, climat, Afrique de l'Ouest, impact, tsé-tsé, trypanosomoses.

\begin{abstract}
Résumé :
Les évolutions démographiques (population humaine multipliée par quatre en 50 ans), les changements climatiques et le développement économique survenus en Afrique de l'Ouest depuis maintenant un siècle ont profondément modifié le peuplement humain et les paysages dans lesquels il s'inscrit. Ces évolutions ne sont pas sans conséquences sur le complexe pathogène des trypanosomoses transmises par les glossines. En l'espace d'un siècle, la limite nord des tsé-tsé s'est déplacée vers le sud, accompagnant (mais à des échelles distinctes) la descente des isohyètes et des foyers de maladie du sommeil, autrefois présents en savane et qui sont actuellement répartis en zone forestière et en zone de mangrove. On met en évidence un impact clair de la pression anthropique sur le recul des glossines du groupe morsitans, vecteurs majeurs des trypanosomoses animales. En revanche, les glossines du groupe palpalis Inotamment Glossina palpalis) s'adaptent aux fortes densités humaines et se maintiennent dans les plus grands centres urbains d'Afrique de l'Ouest, posant d'ailleurs la question du développement de foyers urbains dans les années à venir, comme en témoigne l'exemple récent de Kinshasa. L'étude des facteurs humains /dynamiques de peuplement) et environnementaux (changements climatiques et leurs impacts) dans l'espace et dans le temps, corrélée à celle des trypanosomoses et de leurs vecteurs devra permettre la mise en évidence des combinaisons spatiales capables d'expliquer la présence/absence de la maladie dans le but d'orienter les zones d'intervention médicale et anti-vectorielle. Ce type d'étude pourra être étendu à d'autres pathologies transmises par des vecteurs.
\end{abstract}

\section{INTRODUCTION}

L es évolutions démographiques et le développement économique survenus en Afrique de l'Ouest depuis maintenant un siècle, ont profondément

\footnotetext{
* Institut de Recherche pour le Développement (IRD), UMR 177 IRDCIRAD, Centre International de Recherche Développement sur l'Élevage en zone Subhumide (CIRDES), 01 BP 454, Bobo-Dioulasso, Burkina Faso.

** Centre International de Recherche Développement sur l'Élevage en zone Subhumide (CIRDES), 01 BP 454, Bobo-Dioulasso, Burkina Faso.

**:* Centre Muraz, 01 BP 390, Bobo-Dioulasso, Burkina Faso.

Correspondance : Philippe Solano.

Tél. : + (226) 20985194 - Fax : + (226) 20970942.

E-mail : solano@ird.bf

${ }^{1}$ Texte issu d'une communication à la conférence du CIRDES "Évolutions démographiques et changements climatiques : impacts sur les maladies à transmission vectorielle en Afrique de l'Ouest", 2427 novembre 2008, Ouidah, Bénin.
}

modifié la physionomie du peuplement humain. Tout d'abord du fait de l'accroissement naturel, divergent selon les régions, puis avec les migrations et les déplacements de population, véritables distributeurs du jeu de cartes démographiques (Guengant, 2007). L'augmentation des densités humaines en zone rurale et le développement du fait urbain sont les exemples les plus concrets de cette évolution (OCDE, 1998).

Les changements climatiques ont également contribué à modeler la répartition spatiale des hommes, notamment dans cette partie du monde où les populations tirent l'essentiel de leurs revenus de la terre et du ciel. La sécheresse qui a touché l'Afrique de l'Ouest dans les années 1970-1990 et les migrations de population qu'elle a provoquées en est probablement l'exemple récent le plus probant (Bernus, 1999; L’Hôte \& Mahé, 1996). Une des principales conséquences de ces évolutions démo-climatiques est l'intense mobilité de la popu- 
lation Ouest-Africaine et son impact sur l'environnement. Cette mobilité s'effectue aussi bien des villes vers la campagne, que des pays sahéliens vers les pays côtiers, ou que de l'Afrique vers l'Europe (Robin, 1996). En Afrique de l'Ouest, depuis une vingtaine d'années, on constate qu'elle évolue dans ces modalités d'exécution, car de volontaire (migrations de populations) cette mobilité devient de plus en plus forcée (déplacements de populations) (Lassailly-Jacob et al., 1999). On peut prendre à titre d'exemples la multiplication des conflits comme au Libéria, en Sierra Leone et en Côte-d'Ivoire (UNOCHA, 2003) et l'augmentation du nombre d'accidents climatiques, comme les inondations de septembre 2007 (UNOCHA, 2007).

Les évolutions démo-climatiques ont donc un impact majeur sur l'organisation du peuplement humain, qui lui-même perturbe les niches écologiques favorables aux insectes vecteurs, laissant ainsi l'opportunité aux complexes pathogènes de s'exprimer (Sorre, 1943) et d'évoluer, soit vers l'extinction, soit vers la résurgence et/ou l'épidémisation. Les pathologies à transmission vectorielle évoluent dans l'espace et dans le temps en fonction des dynamiques paysagères impulsées par les besoins des sociétés humaines, assouvis grâce à l'utilisation de techniques de production (Gourou, 1973). Que ce soit pour la bilharziose (Doumenge et al., 1983; Handscumacher, 1992), l'onchocercose (Paris, 1992), le paludisme (Amat-Roze \& Rémy, 1983) ou la trypanosomose (Hervouët \& Laveissière, 1987), les modalités d'exploitation des territoires orchestrées par l'homme jouent un rôle primordial dans la présence/absence de ces maladies et dans leurs différents niveaux de prévalence. Les trypanosomoses humaines africaines (THA ou maladie du sommeil) et animales africaines (TAA ou "nagana") transmises par les glossines (mouches tsétsé) n'échappent pas à cette règle, comme nous allons le montrer.

\section{HISTORIQUE DE LA DISTRIBUTION GÉOGRAPHIQUE DES TRYPANOSOMOSES}

$\mathrm{E}$ n Afrique de l'Ouest, au début du XX ${ }^{\text {ème }}$ siècle, les trypanosomoses ont constitué un frein à l'exploitation des territoires, aussi bien par la ponction démographique infligée aux hommes avec la THA (Jamot, 1933), que par le handicap alimentaire provoqué du fait des TAA (Mornet, 1954). Suite aux actions médicales massives organisées par les équipes mobiles contre la THA en Afrique de l'Ouest (Muraz, 1943), cette endémie fut déclarée "résiduelle" au lendemain des indépendances (Richet, 1962). Cependant, dans les années 1970, probablement du fait d'un relâchement des efforts de lutte et aussi de nouvelles inter- actions hôte-vecteur-parasite, de nombreux foyers historiques (Bouaflé, Daloa, Ouéléssébougou, Dédougou...) et nouveaux (Vavoua, Bonon...) se sont développés (Saliou \& Challier, 1976; Lankoande \& Ouedele, 1982; Duvallet et al., 1979; Solano et al., 2003) pour atteindre dans les années 1990 des niveaux de prévalence parfois comparables à ceux des années 1930. Récemment, une redynamisation des Programmes nationaux a vu le jour sous la bannière de l'OMS, et le nombre de malades a recommencé à diminuer en Afrique Centrale (Simarro et al., 2008). Moins d'informations sont disponibles en Afrique de l'Ouest, en particulier pour des pays comme le Libéria, la Sierra Leone, la GuinéeBissau et le Nigéria. La maladie du sommeil reste bien une maladie "négligée".

En Afrique de l'Ouest, en comparaison au siècle dernier, c'est surtout le changement dans la distribution géographique des cas qui est surprenant. En effet, alors que la THA sévissait principalement dans toute la zone de savane d'Afrique de l'Ouest, avec comme épicentre la Haute-Volta de l'époque, nous avons récemment mis en évidence que de nos jours la THA endémique est présente en zone de forêt dégradée (Côte-d'Ivoire) et de mangrove (Guinée) (Courtin et al., 2008a), mais semble avoir disparu des zones de savane. Quels sont les facteurs qui peuvent expliquer une telle dynamique spatiale, qui se traduit dans la réalité par une évolution des relations entre les trois acteurs du système pathogène (l'homme, la glossine et le trypanosome) et du paysage dans lequel ils s'inscrivent? Nous pensons que les évolutions démo-climatiques jouent un rôle primordial dans ces relations, de la savane burkinabè à la mangrove guinéenne en passant par la forêt mésophile ivoirienne.

\section{PEuplement, PAYSAGE, TRYPANOSOMOSES ET TSÉ-TSÉ} EN AFrique de l'OUeST lorS DE LA COLONISATION

Les premières descriptions effectuées sur le peuplement par les explorateurs militaires coloniaux laissent l'impression d'une Afrique de l'Ouest sous-peuplée, profondément marquée par les conflits et l'insécurité (Gouraud, 1939; Thomann, 1999). L'on peut ainsi observer que les densités de populations en Afrique de l'Ouest établies par le capitaine Binger s'échelonnent de 1 à 25 hab $/ \mathrm{km}^{2}$ (Binger, 1892). Le commerce de la kola, du sel, de l'ivoire et des captifs suit alors le tracé de pistes incertaines qui traversent une végétation luxuriante habitée par une faune sauvage importante et diversifiée.

Dès le début du XX ${ }^{\text {ème }}$ siècle, l'exploitation des territoires nouvellement acquis et à forte potentialité économique était donc confrontée à un problème majeur : le manque de main d'œuvre (Sarraut, 1920). Les principales raisons de cette carence démographique étaient, outre la traite esclavagiste et la ponction humaine infligée par les deux conflits mondiaux, le "fardeau pathologique" que supportaient les Africains (Bado, 
1996). Ce dernier était caractérisé par la prédominance des maladies parasitaires et parmi elles, la maladie du sommeil transmise par les glossines. Les prévalences présentaient de profondes disparités spatiales et dans certaines régions, c'est bien la maladie du sommeil qui modelait le peuplement et par conséquent le paysage, comme par exemple en pays Lobi : "Dans le cercle de Gaoua, la morbidité et la mortalité dues à la trypanosomiase sont impressionnantes. Nous ne parlons pas des quelques cas que nous avons traités au dispensaire de Diébougou, mais des foyers de brousse, des villages contaminés, décimés et abandonnés par leurs occupants effrayés par le fléau qui les frappe et qui fuient pour s'y soustrair' (Jamot, 1933).

La trypanosomose animale n'était pas en reste, notamment sur les abords de la Volta Noire, où le célèbre poste militaire de Koury fut abandonné à cause des Trypanosomoses (Gouzien, 1908; Rouamba et al., 2009). Jusqu'en 1939, les foyers de THA sont localisés en zone de savane (Côte sénégalaise, Casamance, Soudan, HautSénégal-Niger, Nord Dahomey) et des glossines sont capturées jusqu'à Saint-Louis, Djenné, Dori et Niamey (figure 1) (Jamot, 1933). Il faut attendre 1939, Muraz et la création du Service général autonome de la maladie du sommeil (SGAMS), pour voir l'Afrique Occidentale Française (AOF) découpée en secteurs spéciaux et annexes et identifier les premiers grands foyers forestiers ivoiriens et guinéens. Les anglais organisèrent également la lutte dans leurs colonies, mais elle fut plus centrée sur le vecteur (Nash, 1948). C'est ainsi que de 1930 à 1950 on estime à 550000 le nombre de trypanosomés dépistés dans toute l'Afrique de l'Ouest, pour une population totale de 64 millions d'habitants en 1950 (Courtin et al., 2008a).

PEUPLEMENT, PAYSAGE, TRYPANOSOMOSES ET TSÉ-TSÉ EN AFrique DE l'OUest AuJOURD'HuI

Les démographes estiment la population de l'Afrique de l'Ouest à 240 millions d'habitants en 2000, soit une population multipliée par quatre en 50 ans (Guengant, 2007). Cette augmentation des densités humaines, unique dans l'histoire de l'humanité, a profondément modifié le peuplement d'Afrique de l'Ouest, ses paysages, ainsi que la distribution des glossines (figure 2). Aujourd'hui en Afrique de l'Ouest, la limite nord des glossines se situe au niveau de Dakar et de Ouagadougou, soit une descente d'environ 200 kilomètres par rapport aux résultats de piégeages de Bouët-Roubaud du siècle dernier, ce qui explique la disparition de certains foyers historiques, tels que Saint-Louis ou Say (Courtin et al., 2008b). Il est difficile de faire la part des choses entre l'impact de l'anthropisation et celui du climat, par exemple à travers les épisodes de sécheresse des années 1970-1990 (Paturel et al., 1998). En fait, cette action climatique a surtout joué au niveau de la limite nord des glossines, directement par la modification des conditions hygrométriques et de température nécessaires à la survie des glossines (L'Hôte \& Mahé, 1996), indirectement par l'augmentation de la pression anthropique sur la ressource végétale et animale (Boutrais, 1999). La disparition des glossines de la Somone dans le foyer historique de la Petite Côte au Sénégal en est un bon exemple (Laveissière \& Traoré, 1979).

Bien qu'une descente au sud de la limite nord des tsétsé (à l'échelle de l'Afrique de l'Ouest) ait été observée, elle n'explique pas pourquoi aujourd'hui la THA ne sévit plus qu'en zone de forêt et de mangrove et a disparu des zones de savane, alors que les tsé-tsé y existent encore et qu'elles y transmettent les trypanosomoses animales (Burkina Faso, Mali...).

Une autre conséquence, attribuable, elle, directement à l'impact de la pression anthrophique, est l'observation du recul progressif dans le bassin de Mouhoun (ex Volta-Noire) au Burkina Faso de Glossina morsitans submorsitans, espèce savanicole (Bouyer, 2006). Ce recul est extrapolable à l'ensemble du pays comme le montrent des résultats récents (Rayaissé et al., 2009). Cette espèce est inféodée à la faune sauvage, sa principale source de nourriture, ce qui la rend particulièrement sensible à l'anthropisation destructrice de cette faune sauvage.

L'anthropisation modifie quantitativement mais aussi qualitativement le peuplement des glossines. En zone forestière ivoirienne, c'est le défrichement des forêts pour les transformer en plantations (café, cacao en zone de forêt) qui va provoquer le réveil de la THA via une progressive dominance du vecteur $G$. palpalis qui ne rechigne pas à se nourrir sur l'homme, aux dépens des espèces forestières zoophiles du groupe fusca qui, elles, disparaissent (Laveissière \& Hervouët, 1988).

Il est maintenant connu que, au contraire des glossines de savane du groupe morsitans, G. palpalis s'adapte si bien à l'homme que des foyers urbains de THA peuvent exister (Louis et al., 2003; Courtin et al., 2005), et que l'on observe de fortes densités de cette espèce dans la plupart des grands centres urbains d'Afrique de l'Ouest (Abidjan, Conakry, Bamako, Dakar), et même d'Afrique Centrale.

Lorsqu'on regarde la carte de l'actuel peuplement et du paysage d'Afrique de l'Ouest, on comprend l'incroyable évolution parcourue en l'espace d'un siècle (figure 2). L'anthropisation provoque en savane : 1) la dégradation du couvert végétal d'interfluve et ripicole indispensable à la survie des glossines; 2) la quasi-disparition de la faune sauvage indispensable à la présence des glossines de savane, et favorable aux fortes densités de glossines riveraines; 3) le développement des aménagements hydrauliques (pompes, puits modernes). En combinant ces facteurs historiques et actuels (stérilisation du réservoir humain de trypanosomes par les équipes médicales, impact environnemental de l'anthro- 


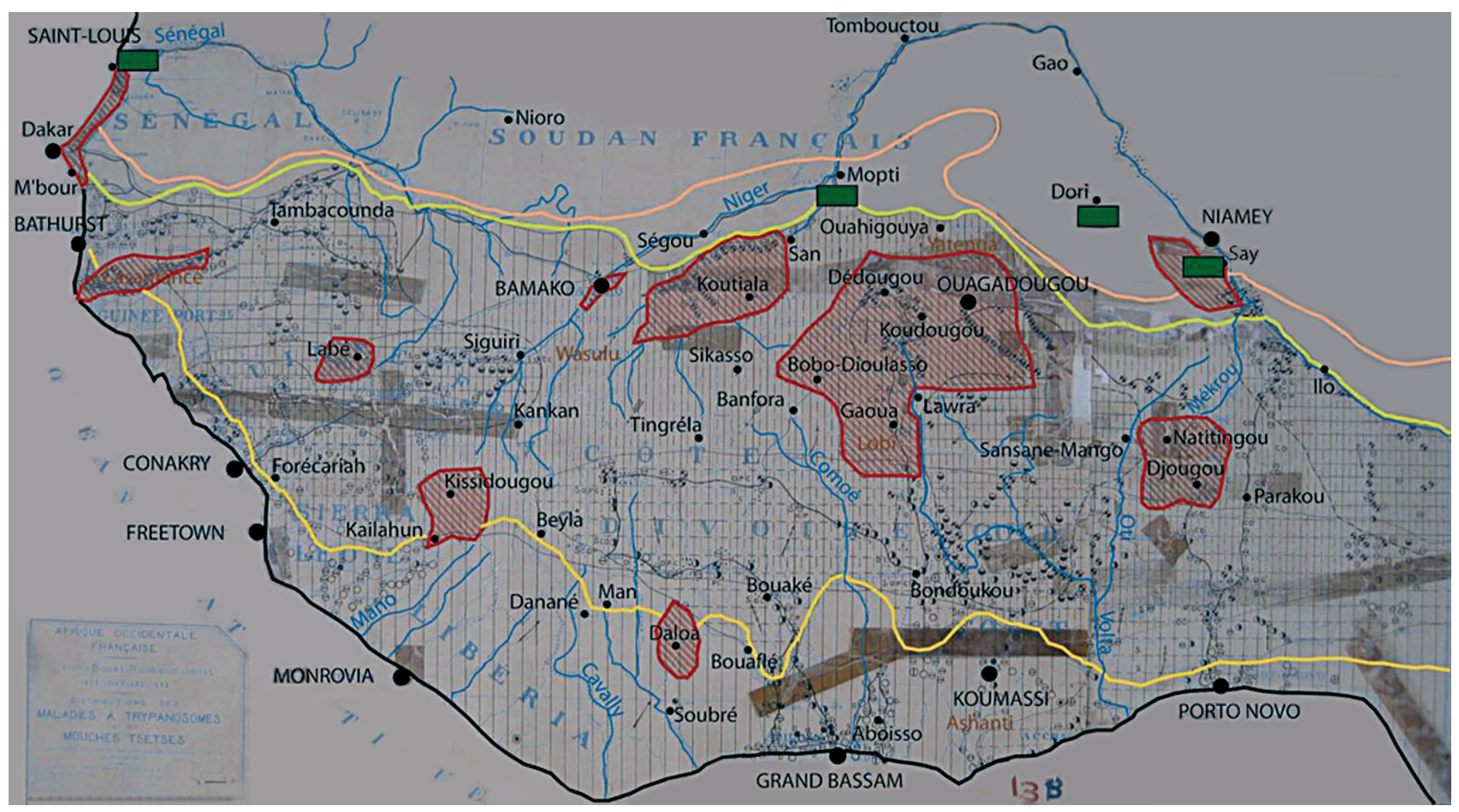

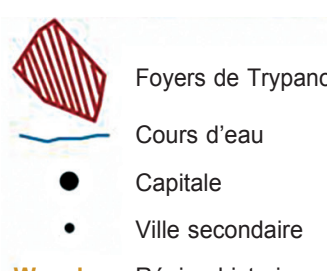

Wasulu Région historique
Capture septentrionale de Glossina palpalis (1906-1916)

Limite des savanes soudaniennes et guinéennes

Limite de la végétation boisée des cours d'eau

Limite de la Grande Forêt

Dressé, dessiné, héliogravé et imprimé au service Géographique de l'AOF, 1935

Fig. 1. - Peuplement, paysage, glossine et trypanosomose humaine africaine (THA) dans la première moitié du XXème siècle.

Source : Afrique Occidentale Française, missions Bouët-Roubaud-Jamot (1906-1916 ; 1932-1935) - "Distribution des maladies à trypanosomes et des mouches tsé-tsé". Échelle 1/2 500000.

Nota bene: les bandes gris/marron n'ont pas de signification scientifique; il s'agit de traces de papier collant au dos de ce document ancien.

pisation, et diminution du contact homme-glossine), l'on a un début de réponse à la question de la disparition de la THA des zones de savane et de sa non-réapparition alors que les glossines (riveraines) y sont toujours présentes, et qu'elles sont toujours capables de transmettre les TAA aux animaux domestiques, via un contact majoritairement localisé aux points d'abreuvement (de la Rocque et al., 1999). Il est toutefois très probable que d'autres facteurs issus de ces nouvelles interactions homme-vecteur-environnement encore mal connus et restant à être identifiés, contribuent à cette situation.

PEUPlement, PAYSAGE, TRYPANOSOMOSES ET TSÉ-TSÉ EN Afrique De L'OUest DEMAIN?

La remarquable étude intitulée West Africa Long-Term Perspective Study (WALTPS) détermine quatre grandes zones de peuplement en Afrique de l'Ouest (OCDE, 1998) et leur évolution respective dans les années à venir. Au vu de ces observations, et de l'estimation d'un chiffre de 617 millions d'habitants en 2050 (Guengant, 2007), on pourrait intuitivement penser que les glossines et les trypanosomoses vont disparaittre d'ellesmêmes dans les années à venir, conséquence directe de l'augmentation des densités humaines et de leur impact environnemental, ce qui serait évidemment d'une grande aide aux programmes internationaux récemment initiés de lutte contre ces endémies : Élimination de la THA par l'OMS; Élimination des tsé-tsé et des trypanosomoses, Union Africaine-PATTEC (Pan African Tsetse and Trypanosomosis Eradication Campaign). Toutefois, quelques points méritent d'être soulignés pour tempérer des pronostics un peu précipités. Si évaluer le poids d'une population dans le futur est un exercice complexe, pronostiquer sa future distribution, du fait du jeu des migrations et des déplacements de population l'est encore plus. La crise ivoirienne récente nous rappelle combien l'exercice de la pro- 


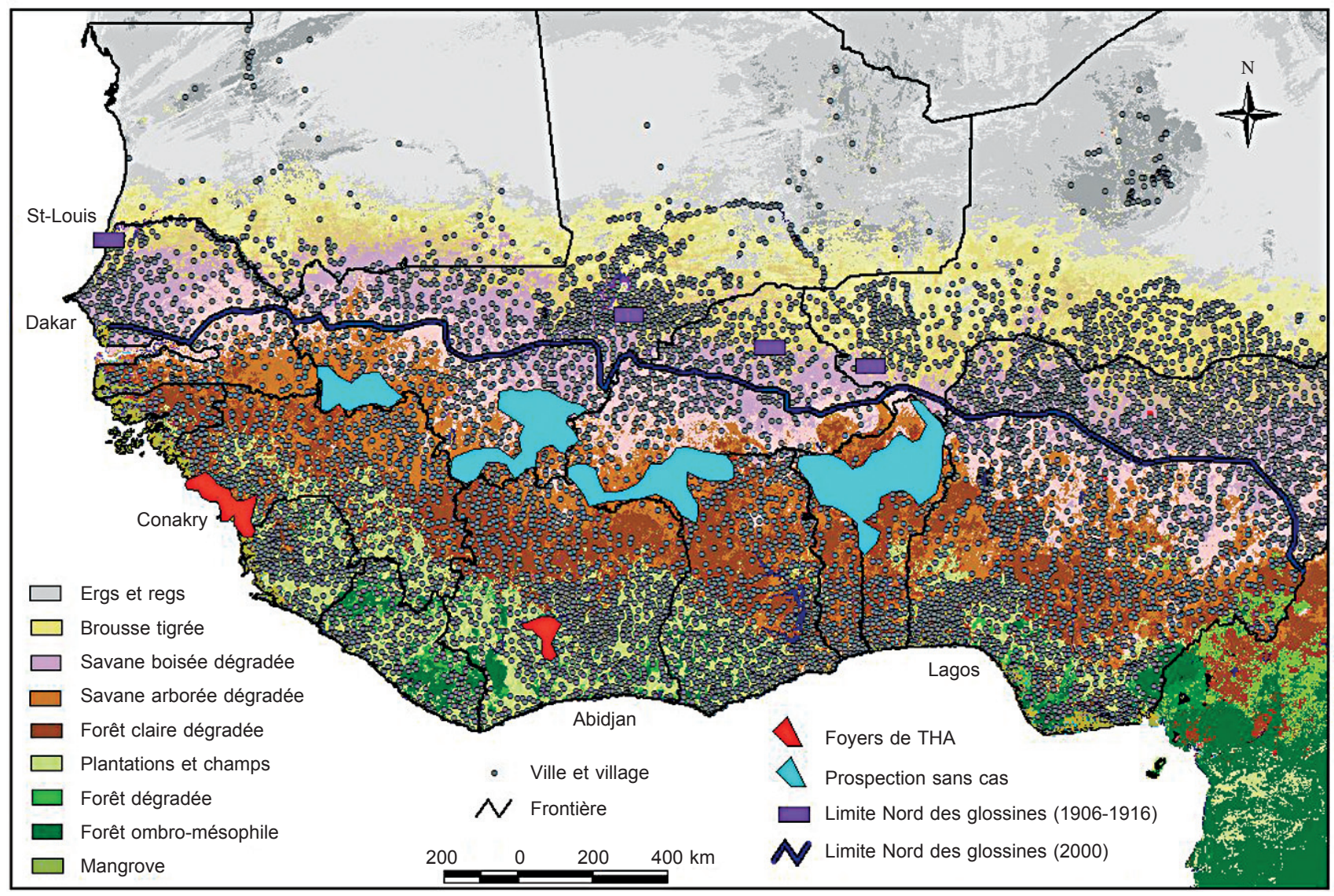

Fig. 2. - Peuplement, paysages, glossines et trypanosomose humaine africaine (THA) aujourd'hui.

Source : Bouët-Roubaud (1906-1916), PAAT-IS (2000), IRD/CIRDES/OMS/PAAT (2000-2008), Landcover 2000 , Maproom 1992.

jection du peuplement est difficile, car avec un total de 1000000 de personnes déplacés, réfugiés et rapatriés, cette projection est aujourd'hui biaisée (UNOCHA, 2003). Notons également que l'instabilité politique est évidemment défavorable à l'organisation de toute lutte médicale et/ou entomologique, et aussi que le développement de pathologies y est bien souvent associé. Or les perturbations socio-politiques, en partie liées à la croissance démographique, risquent de devenir de plus en plus fréquentes, du fait de la lutte pour les ressources économiques, foncières et hydrologiques : "L'Afrique de l'Ouest, région de peuplement accéléré, sera, pendant une longue période, une région en déséquilibre" (OCDE, 1998).

Dans un tel contexte d'insécurité, les modalités d'élevage évolueront probablement aussi, avec notamment une augmentation de l'élevage urbain et péri-urbain comme c'est déjà le cas, et des difficultés croissantes liées au nomadisme et aux conflits agriculteurs-éleveurs (Fall et al., 2004; Boutrais, 1999). Ces évolutions ne seront pas sans conséquences sur la distribution spatiale et les schémas épidémiologiques des trypanosomoses animales.
Les projections climatiques réalisées notamment par le GIEC n'incluent pas ou peu l'Afrique de l'Ouest du fait du peu d'informations disponibles, il est donc actuellement difficile de se faire une idée exacte de l'évolution climatique pour cette région. Cependant, on sait que le réchauffement du globe, en partie lié à l'effet de serre, pourrait être de $+2^{\circ} \mathrm{C}$ à $+6^{\circ} \mathrm{C}$. Il est donc probable que certains processus connus lors de réchauffements passés vont se reproduire, en particulier une augmentation de l'intensité de la mousson guinéenne (Morel, 2006). Outre l'augmentation ou la diminution de pluviométrie, ce sont surtout les modalités d'application de cette pluviométrie qui importent : le centre de la Zone de Convergence Intertropicale (ZCIT) sera-t-il situé plus au Nord ou plus au Sud? La ZCIT sera-t-elle plus ou moins large? Les phénomènes climatiques qui se dérouleront à l'intérieur de cette ZCIT seront-ils plus intenses? Les réponses à toutes ces questions diffèrent selon les modèles de projections (d'Orgeval, 2008). La qualité des réponses à ces questions est primordiale, car si les démographes veulent évaluer le poids de la population future d'Afrique de l'Ouest, et les géographes sa distribution et le paysage dans lequel elle s'inscrira, ils 
se doivent de prendre en compte l'évolution du climat en général, et de la pluviométrie en particulier.

Enfin, nous venons d'observer en Afrique de l'Ouest, deux situations contrastées sur l'impact de ces facteurs démographiques et climatiques sur la distribution des tsé-tsé :

1 - Nous confirmons à l'échelle du Burkina Faso que les glossines savanicoles du groupe morsitans résistent très mal à l'augmentation des densités humaines et ses effets (notamment le braconnage, Rayaisse et al., 2009), et il est probable qu'elles soient amenées à jouer des rôles économiques moins importants dans les années à venir, confirmant l'étude de Reid et al., 2000 à l'échelle de l'Afrique.

2 - En revanche, de plus en plus d'observations directes confirment les très bonnes capacités d'adaptation des espèces riveraines du groupe palpalis (notamment $G$. palpalis en Afrique de l'Ouest, et G. fuscipes en Afrique Centrale) aux fortes densités humaines, et leur survie dans des grands centres urbains où elles transmettent les Trypanosomoses, en particulier la THA (exemple récent de l'épidémie à Kinshasa, Ebeja et al., 2003) : Les causes de ces adaptations sont peut-être à rechercher dans la manière dont les populations de ces espèces sont structurées, et dans leur éclectisme alimentaire. Des observations récentes (Kubi et al., 2006) suggèrent également que des tsé-tsé "stressées" sont beaucoup plus dangereuses en terme de capacité de transmission, ce qui pourrait effectivement être le cas lorsque les conditions biotiques et abiotiques (milieu urbain) sont difficiles.

\section{CONCLUSION}

D u début du XX ${ }^{\text {ème }}$ siècle à aujourd'hui, l'Afrique de l'Ouest a connu un peuplement rapide dans un contexte de déficit hydrique global. Les conséquences de ces évolutions démo-climatiques sur le paysage, corrélées aux résultats de la lutte médicale et entomologique, ont profondément modifié la géographie des glossines et des foyers de THA.

Aujourd'hui, contrairement à la situation qui prévalait au début du XX ${ }^{\text {ème }}$ siècle, l'aire de distribution des glossines s'est rétractée et leur limite nord de distribution a glissé vers le sud, accompagnant (mais à des échelles bien distinctes) la descente des isohyètes, des hommes et des foyers de THA, sans qu'un lien de cause à effet direct ne puisse être directement attribué (en zone de savane les glossines sont toujours présentes mais la THA semble en avoir disparu). L'Afrique de l'Ouest est une région de peuplement accéléré qui s'effectue dans un contexte de variabilité climatique encore mal déterminé

Si l'évolution démographique est prévisible, la dynamique de peuplement l'est beaucoup moins. Non seu- lement parce que les conflits pour les ressources remettent constamment en question les prévisions, mais aussi parce que les caractéristiques du changement climatique reste encore une grande inconnue, alors que ce dernier influe directement sur les possibilités de peuplement. Deux scénarios extrêmes sont envisageables, le premier se caractérise par une multiplication des conflits et des déplacements de population face à une diminution des ressources (baisse de la pluviométrie, accidents climatiques) et à une augmentation des besoins (croissance démographique). Le second se caractérise par une augmentation des ressources (augmentation de la pluviométrie) et une augmentation des besoins (croissance démographique).

Ces évolutions ont bien évidemment des impacts fondamentaux sur le système pathogène des trypanosomoses, mais aussi sur celui d'autres pathologies à transmission vectorielle. Nous proposons, à travers un besoin de multidisciplinarité incluant, à côté des entomologistes, géographes et parasitologistes, des démographes, des climatologues et des modélisateurs, d'accentuer les recherches sur les projections futures de la distribution des tsé-tsé et des trypanosomoses. L'étude des facteurs humains et environnementaux dans l'espace et dans le temps, corrélée à celle des Trypanosomoses et de ses vecteurs devra permettre de mettre en évidence des combinaisons spatiales capables d'expliquer la présence/absence de la maladie. Cette mise en évidence des combinaisons de facteurs et leurs connaissances approfondies doit permettre de proposer des scénarios d'épidémisation/extinction qui serviront d'aide à la décision (OMS, PATTEC) pour prioriser les zones d'intervention médicale et de lutte anti-vectorielle.

\section{REMERCIEMENTS}

es auteurs remercient le Pr G. Duvallet pour ses
conseils et encouragements. F.C. est soutenu par
le projet FSP-REFS du MAEE.
RÉFÉRENCES

Amat-Roze J.M. \& Rémy G. Paysage épidémiologique du paludisme dans l'espace ivoiro-voltaïque, in : De l'épidémiologie à la géographie humaine. Doumenge J.P. (ed.), CNRS/CEGET, travaux et documents de géographie tropicale, 1983, 48, 97-107.

BADO J.P. Médecine coloniale et grandes endémies en Afrique (1900-1960), lèpre, trypanosomiase humaine et onchocercose. Karthala, Paris, 1996, 428 p.

Bernus E. Exodes tous azimuts en zone sahélo-saharienne, in : Déplacés et réfugiés, la mobilité sous contrainte. Lassailly-Jacob V. (ed.), IRD, Collection colloques et séminaires, 1999, 195-208. 
Binger L.G. Du Niger au Golfe de Guinée, par le pays de Kong et le Mossi (1887-1889). Hachette, Paris, 1892, 929 p.

Boutrais J. Les éleveurs, une catégorie oubliée de migrants forcés, in : Déplacés et réfugiés, la mobilité sous contrainte. Lassailly-Jacob V. (ed.), IRD, Collection colloques et séminaires, 1999, 161-192.

BOUYer J. Écologie des glossines du Mouhoun au BurkinaFaso : intérêt pour l'épidémiologie et le contrôle des trypanosomoses africaines. Thèse en biologie, Université de Montpellier II, 2006, 204 p.

Courtin F., Dupont S., Zeze D.G., Jamonneau V., Sane B., Coulibaly B., Cuny G. \& Solano P. Trypanosomose humaine africaine : transmission urbaine dans le foyer de Bonon (Côte-d'Ivoire). Tropical Medicine and International Health, 2005, 10 (4), 340-346.

Courtin F., Jamonneau V., Duvallet G., Garcia A., Coulibaly B., Doumenge J.P., Cuny G. \& Solano P. Sleeping sickness in West Africa (1906-2006): changes in spatial repartition and lessons from the past. Tropical Medicine and International Health, 2008a, 13 (3), 334-344.

Courtin F., Jamonneau V., Duvallet G., Camara M., Kaba D. \& Solano P. Un siècle de "trypano" en Afrique de l'Ouest. Bulletin de Société de Pathologie Exotique, 2008b, 101 (3), 287-289.

de la Rocque S., Bengaly Z., Michel J.F., Solano P., Sidibé I. \& CuIsance D. Importance des interfaces spatiales et temporelles entre les bovins et les glossines dans la transmission de la trypanosomose animale en Afrique de l'Ouest. Revue d'élevage et de médecine vétérinaire des pays tropicaux, 1999, 52, 215-222.

D'Orgeval T. Impact du changement climatique sur la saison des pluies en Afrique de l'Ouest : que nous disent les modèles de climat actuels? Sécheresse, 2008, 19, 79-85.

Doumenge J.P., Cheung C., Villenave D. \& Guérin B. Intérêt et limites d'une cartographie des schistosomiases humaines dans le monde, in : De l'épidémiologie à la géographie humaine. Doumenge J.P. (ed.), CNRS/CEGET, travaux et documents de géographie tropicale, 1983, 48, 169-176.

Duvallet G., Stanghellini A., Saccharin C. \& Vivant J.F. Human trypanosomiasis focus of Vavoua (Ivory Coast), a clinical, parasitological and sero-immunological survey. Médecine tropicale, 1979, 39, 517-526.

Ebeja A.K., Lutumba P., Molisho D., Kegels G., Miaka mia Bilenge C. \& Boelaert M. Sleeping sickness in the region of the town of Kinshasa: a retrospective analysis during the surveillance period 1996-2000. Tropical Medecine and International Health 2003, 8, 949-955.

Fall A., Ba Diao M., Bastianelli D. \& Nianogo A. La gestion concertée et durable des filières animales urbaines, in : Développement durable de l'agriculture urbaine en Afrique francophone, Enjeux, concepts et méthodes. Smith B., Moustier P., Mougeot L. \& Fall A. (eds), CIRAD/CRDI, 2004, 115141.

Gouraud H. Au Soudan. Éditions Pierre Tisné, Paris, 1939, $253 \mathrm{p}$.

Gourou P. Pour une géographie humaine. Flammarion, Paris, 1973, 388 p.

Gouzien P. La maladie du sommeil dans le Haut-Sénégal et Niger. $\mathrm{n}^{\circ}$ 490/Document Technique OCCGE, 1908, 25 p.
GuENGANT J.P. La démographie africaine entre convergences et divergences, in : L'Afrique face à ses défis démographiques, un avenir incertain. Ferry B. (Eds), AFD-CEPEDKarthala, Paris, 2007, 25-121.

Handschumacher P. Les fondements géographiques de la bilharziose à Richard-Toll, in : L'eau et la santé dans les contextes du développement : volet Sénégal. Éditions de l'ORSTOM, 1992, 16 p.

HervouËT J.P. \& Laveissière C. Facteurs humains de la maladie du sommeil. La Medicina Tropicale Nella Cooperzione Allo Sviluppo, 1987, 3 (2), 72-78.

Jамот E. Contribution à l'étude de la maladie du sommeil en Afrique Occidentale Française (Ouagadougou). n 492/ Document Technique OCCGE, 1933.

Kubi C., Van Den Abbeele J., De Deken R., Marcotty T., Dorny P. \& VAN DEN Bossche P. The effect of starvation on the susceptibility of teneral and non teneral tsetse flies to trypanosome infection. Medical and Veterinary Entomology, 2006, 20 (4), 388-392.

LANKOANDE S.F. \& OUEDELE M.N. La trypanosomiase humaine dans le foyer de la Volta-Noire (Dédougou-Boromo) Haute-Volta. Médecine d'Afrique noire, 1982, 29 (2), $157-$ 161.

Lassailly-Jacob V., Marchal J.Y. \& Quesnel A. Déplacés et réfugiés, la mobilité sous contrainte. Éditions IRD, Collection colloques et séminaires, 1999, 504 p.

Laveissière C. \& Traoré T. Enquête entomologique dans le foyer de trypanosomiase humaine de la Somone (République du Sénégal, mai 1979). Centre Muraz, rapport de mission $n^{\circ}$ 7166/79, Document Technique OCCGE, 1979, 12 p.

LAveissière C. \& HervouËT J.P. Épidémiologie et contrôle de la trypanosomiase humaine en Afrique de l'Ouest. Éditions de l'ORSTOM, Collection didactique, 1988, 157 p.

L'Hôte Y. \& Mahé G. Afrique de l'Ouest et Centrale, précipitations moyennes annuelles (période 1951-1989), départements eaux continentales, laboratoire de cartographie appliquée, ORSTOM, 1996 : http://www.cartographie. ird.fr/images/pluvio_afrique/pluvio3.gif

Louis F.J., Mia Bilenge C.M., Simarro P., Kande Betu Ku Meso V., LuCAS P. \& Jannin J. Trypanosomose humaine africaine en milieu urbain : une problématique émergente? Bulletin de la Société de Pathologie Exotique, 2003, 96 (3), 205-208.

MAHÉ G. \& Olivry J.C. Changements climatiques et variations des écoulements en Afrique occidentale et centrale, du mensuel à l'interannuel. Hydrology for the Water Management of Large River Basins, 1991, 201, 163-171.

Morel A. "Le désert avance...", in : L’Afrique des idées reçues, Courade G. (ed.). Belin, Paris, 2006, 106-111.

MORNET P. Les trypanosomes pathogènes de l'AOF, considérations sur leur répartition, leur fréquence, le taux d'infestation des animaux domestiques. Éditions BPITT/CCTA, Comité scientifique international de recherches sur les trypanosomiases, cinquième réunion, Prétoria, 13-17 septembre 1954, 709-720.

Muraz G. Lutte contre la maladie du sommeil en AOF et au Togo. Académie des Sciences Coloniales 1943, 8, 593622 
Nash T.A.M. Tsetse flies in British West Africa. Published for the Colonial Office by HMSO, London, 1948, 77 p.

OCDE. Pour préparer l'avenir de l'Afrique de l'Ouest, une vision à l'horizon 2020. Cour J.M. \& Snrech S. (eds). OCDE, Paris, 1998, 157 p.

PARIS F. De l'onchocercose à la "géo-oncho-graphie", in : Dynamiques des systèmes agraires : la santé en société : regards et remèdes. Blanc-Pamard C. (ed.). ORSTOM, Collection colloques et séminaires, 1992, 58-85.

Paturel J.E., Servat E., Delattre M.O. \& Lubes-Niel H. Analyse de séries pluviométriques de longue durée en Afrique de l'Ouest et Centrale non sahélienne dans un contexte de variabilité climatique. Hydrological Sciences, 1998, 43 (6), 937-946.

Picheral H. Complexes et systèmes pathogènes : approche géographique, in : De l'épidémiologie à la géographie humaine. Doumenge J.P. (eds), CNRS/CEGET, travaux et documents de géographie tropicale, 1983, 48, 5-22.

Rayaissé J.B., Courtin F., Akoudjim M., Cesar J. \& Solano P. Influence de l'anthropisation sur la végétation locale et l'abondance des tsé-tsé au sud du Burkina Fazo. Parasite, 2009, 16 (1), 21-28.

Reid S.R., Kruska R.L., Deichmann U., Thornton P.K. \& Leak S. Human population growth and the extinction of the tsetse fly. Agriculture, Ecosystem and Environment, 2000, 77, 227-236.

RicheT P. La trypanosomiase résiduelle. OMS, comité des experts en trypanosomiase, Genève, 18-23 juin 1962, 30 p.

Robin N. Atlas des migrations ouest-africaines vers l'Europe, 1985-1993. Éditions de l'ORSTOM, 1996, 108 p.

Rouamba J., Jamonneau V., Sidibé I., Solano P. \& Courtin F. Impact de la dynamique de peuplement sur la distribution des glossines dans la boucle du Mouhoun (Burkina Faso). Parasite, 2009, 16 (1), 11-19.

Saliou P. \& Challier A. Compte rendu de mission dans le foyer de maladie du sommeil de Bouaflé (Côte-d'Ivoire), avril 1976 : étude de la situation de l'endémie et propositions d'un programme de lutte. Centre Muraz, rapport de mission $n^{\circ}$ 6186/76, Document Technique OCCGE, 1976, $15 \mathrm{p}$.

SARRAUT A. Interpellations sur les ressources coloniales. Journal Officiel de la République Française, 1920, 12 p.

SimarRo P., JANNIN J. \& CATTAND P. Eliminating human african trypanosomiasis: where do we stand and what comes next? PloS Medicine 2008, 5, 174-180.

Solano P., Kone A., Garcia A., Sane B., Michel V., Michel J.F., Coulibaly B., Jamonneau V., Kaba D., Dupont S. \& FouRNET F. Rôle des déplacements des malades dans l'épidémiologie de la trypanosomose humaine africaine dans le foyer de Bonon, Côte d'Ivoire. Médecine tropicale, 2003, 63, 577-582.

SORRE M. Les fondements biologiques de la géographie humaine, essai d'une écologie de l'homme. Armand Colin, Paris, 1943, $440 \mathrm{p}$.

Thomann G. Carnets de route en Côte-d'Ivoire (1893-1902). Éditions SEPIA, Saint-Maur-des-fossés, 1999, 232 p.

UNOCHA West Africa-Situation map, Regional support office, October 2003: http://www.reliefweb.int/rw/fullMaps_Af.nsf/
luFullMap/74DDDC3E87A7564D85256DD0005AC1FD/\$Fil/ rw_wasitmao311003.pdf?OpenElement

UNOCHA West Africa-Floods, September 2007: http:// www.reliefweb.int/rw/fullMaps_Af.nsf/luFullMap/E96B5A7 BF8FC89758525736E0065AF3E/\$File/ocha_FL_afr071004.pd f?OpenElement

Reçu le 13 décembre 2008 Accepté le 9 janvier 2009 